\title{
TI.100.1
}

\section{InCommon Federation Security Incident Handling}

- PDF: InCommon Security Incident Framework.pdf

- HTML: InCommon Federation Security Incident Handling.htm

\section{More Information}

\begin{tabular}{|l|l|}
\hline Repository ID & TI.100.1 \\
\hline Persistent URL & http://doi.org/10.26869/TI.100.1 \\
\hline Title & InCommon Federation Security Incident Handling \\
\hline Authors & Nicole Roy \\
\hline Sponsor & InCommon Steering Committee \\
\hline Review & \\
\hline Status & preserve \\
\hline Publish Date & January 30,2017 \\
\hline DOI & $10.26869 / T I .100 .1$ \\
\hline Signature & \\
\hline Deprecated & Yes \\
\hline Future Review & March 1.2019 \\
\hline Supersedes & none \\
\hline Format & \\
\hline Related Docs & \\
\hline Development Location & https://github.internet2.edu/internet2/inc-incident-handling-fx \\
\hline IP Framework & \\
\hline Subject Tags & federation, trust, incommon \\
\hline Notes & \\
\hline & \\
\hline
\end{tabular}

